\title{
Universal Scaling of Nonequilibrium Transport in the Kondo Regime of Single Molecule Devices
}

\author{
G D. Scott \\ Rice University \\ Z K. Keane \\ Rice University \\ Jacob W. Ciszek \\ Loyola University Chicago, jciszek@luc.edu \\ J M. Tour \\ Rice University \\ D Natelson \\ Rice University
}

Follow this and additional works at: https://ecommons.luc.edu/chemistry_facpubs

Part of the Chemistry Commons

\section{Recommended Citation}

Scott, GD, ZK Keane, JW Ciszek, JM Tour, and D Natelson. "Universal scaling of nonequilibrium transport in the kondo regime of single molecule devices" in Physical Review B 79, 2009.

This Article is brought to you for free and open access by the Faculty Publications and Other Works by Department at Loyola eCommons. It has been accepted for inclusion in Chemistry: Faculty Publications and Other Works by an authorized administrator of Loyola eCommons. For more information, please contact ecommons@luc.edu. cc) (i) $\ominus$

This work is licensed under a Creative Commons Attribution-Noncommercial-No Derivative Works 3.0 License. (c) American Physical Society, 2009. 


\title{
Universal scaling of nonequilibrium transport in the Kondo regime of single molecule devices
}

\author{
G. D. Scott, ${ }^{1, *}$ Z. K. Keane, ${ }^{1}$ J. W. Ciszek, ${ }^{2,3}$ J. M. Tour, ${ }^{3}$ and D. Natelson ${ }^{1,4}$ \\ ${ }^{1}$ Department of Physics and Astronomy, Rice University, 6100 Main Street, Houston, Texas 77005, USA \\ ${ }^{2}$ Department of Chemistry, Loyola University Chicago, 1068 W. Sheridan Road, Chicago, Illinois 60626, USA \\ ${ }^{3}$ Department of Chemistry, Rice University, 6100 Main Street, Houston, Texas 77005, USA \\ ${ }^{4}$ Department of Electrical and Computer Engineering, Rice University, 6100 Main Street, Houston, Texas 77005, USA
}

(Received 13 February 2009; published 9 April 2009)

\begin{abstract}
Scaling laws and universality are often associated with systems exhibiting emergent phenomena possessing a characteristic energy scale. We report nonequilibrium transport measurements on two different types of single molecule transistor devices in the Kondo regime. The conductance at low bias and temperature adheres to a scaling function characterized by two parameters. This result, analogous to that reported recently in semiconductor dots with Kondo temperatures two orders of magnitude lower, demonstrates the universality of this scaling form. We compare the extracted values of the scaling coefficients to previous experimental and theoretical results.
\end{abstract}

DOI: $10.1103 /$ PhysRevB.79.165413

Scaling theories, most notably of the power-law form, characterize a diverse array of naturally occurring phenomena. Seemingly unrelated systems with widely differing microscopic details can be described by relations with identical scaling exponents when the underlying dynamics are similar. This property, known as universality, is relevant when considering systems driven out of equilibrium by some perturbation. In a system with an emergent characteristic energy scale, examining the system response as a function of the perturbation scaled relative to that energy can reveal such universality.

The many-body Kondo state in quantum dots, as observed in a variety of microscopic implementations, ${ }^{1-6}$ is an example of such a system. In these devices itinerant electrons in source and drain leads are coupled via tunneling barriers to a single magnetic impurity. At zero source-drain bias $(V)$, below an emergent Kondo energy scale, $k_{B} T_{K}$, the local moment of the impurity is screened by the conduction electrons. As a result of the screening process the (zero-bias) conductance, $G$, is enhanced at low temperatures $\left(T<T_{K}\right)$. It has been documented that $G(T, V \approx 0)$ in the equilibrium Kondo regime is well described by a universal temperature dependence. ${ }^{7-9}$ A nonzero applied bias $(|V|>0)$ drives the system into the nonequilibrium Kondo regime. ${ }^{10-12} G(T, V)$ exhibits a resonance peak centered at $V=0$. Theoretical treatments of idealized quantum dots have argued that $G(T, V)$ in the single channel Kondo state may be described by an analogous function in bias voltage and temperature. However, there is discussion regarding the energy scales and order to which this scaling will hold as well as the number of system specific coefficients required, their expected values, and the universality of these coefficients. ${ }^{13-18}$ Recent experiments by Grobis et al. ${ }^{19}$ have shown that $G(T, V)$ measured in a single channel GaAs quantum dot $\left(T_{K} \approx 0.3 \mathrm{~K}\right)$ in the nonequilibrium regime is well described by a universal scaling function with two scaling parameters.

In this paper we test the universality of these results, applying the analogous analytical approach to 29 moleculebased devices with Kondo temperatures ranging from 34 to $155 \mathrm{~K}$. We find that the conductances of single molecule transistors (SMTs) containing either $\mathrm{C}_{60}$ (Refs. 6, 20, and 21) or a transition metal complex ${ }^{22}$ are accurately described by the same scaled parameters as the GaAs dot. ${ }^{19}$ We confirm the quadratic voltage and temperature dependence of $G$ in the low energy limit. The values of the extracted scaling coefficients are quite consistent throughout the ensemble. We discuss possible explanations for the systematic differences between our coefficients and those inferred from previous experiments ${ }^{19}$ and theoretical model predictions.

Sample fabrication begins with e-beam lithographic patterning of gold nanoconstrictions with minimum widths between 90 and $120 \mathrm{~nm}$ in arrays on an $n^{+} \mathrm{Si}$ substrate with a $200 \mathrm{~nm} \mathrm{SiO}{ }_{2}$ insulating layer. Immediately prior to being placed in a cryostat, the chip is cleaned and then treated with the molecules that are to be utilized as the active elements, as described previously. ${ }^{6,22}$ Each device in an array can be contacted individually using an attocube system cryogenic probe station with a base temperature of $\sim 1.8 \mathrm{~K}$. Electromigration in a cryogenic environment is used to create a small gap in the nanowire, ideally allowing a molecule to bridge the broken ends of the wire that will subsequently function as source and drain electrodes [Figs. 1(a) and 1(b)]. ${ }^{23} \mathrm{C}_{60}$ and a Cu-containing transition metal complex (bis(2,5-di[2]pyridyl-3,4-dithiocyanto-pyrrolate)Cu(II)) (“complex 1") were independently utilized as the active elements of our SMTs. Both $\mathrm{C}_{60}$ (Refs. 6, 20, and 21) and complex 1 (Refs. 22 and 24) have been successfully used as active elements in past investigations of break junction devices.

Differential conductance, $d I / d V$, was measured as a function of $V$ using quasi-dc lock-in techniques with an rms excitation voltage of $0.75 \mathrm{mV}$ at $19.149 \mathrm{~Hz}$. Postelectromigration transport properties were consistent with previous reported frequencies of occurrence. ${ }^{24}$ A pronounced zerobias conductance peak was observed in approximately $20 \%$ of devices, approximately one third of which were sufficiently stable at relevant biases and temperatures to enable a complete analysis. The prototypical behavior of the single channel spin- $\frac{1}{2}$ Kondo effect is exhibited by a decreasing amplitude and broadening of the Kondo resonance as the temperature is increased. The temperature dependence of the amplitude has been well described by an empirical form ${ }^{7}$ derived from a fit to the renormalization group analysis, ${ }^{11}$ 

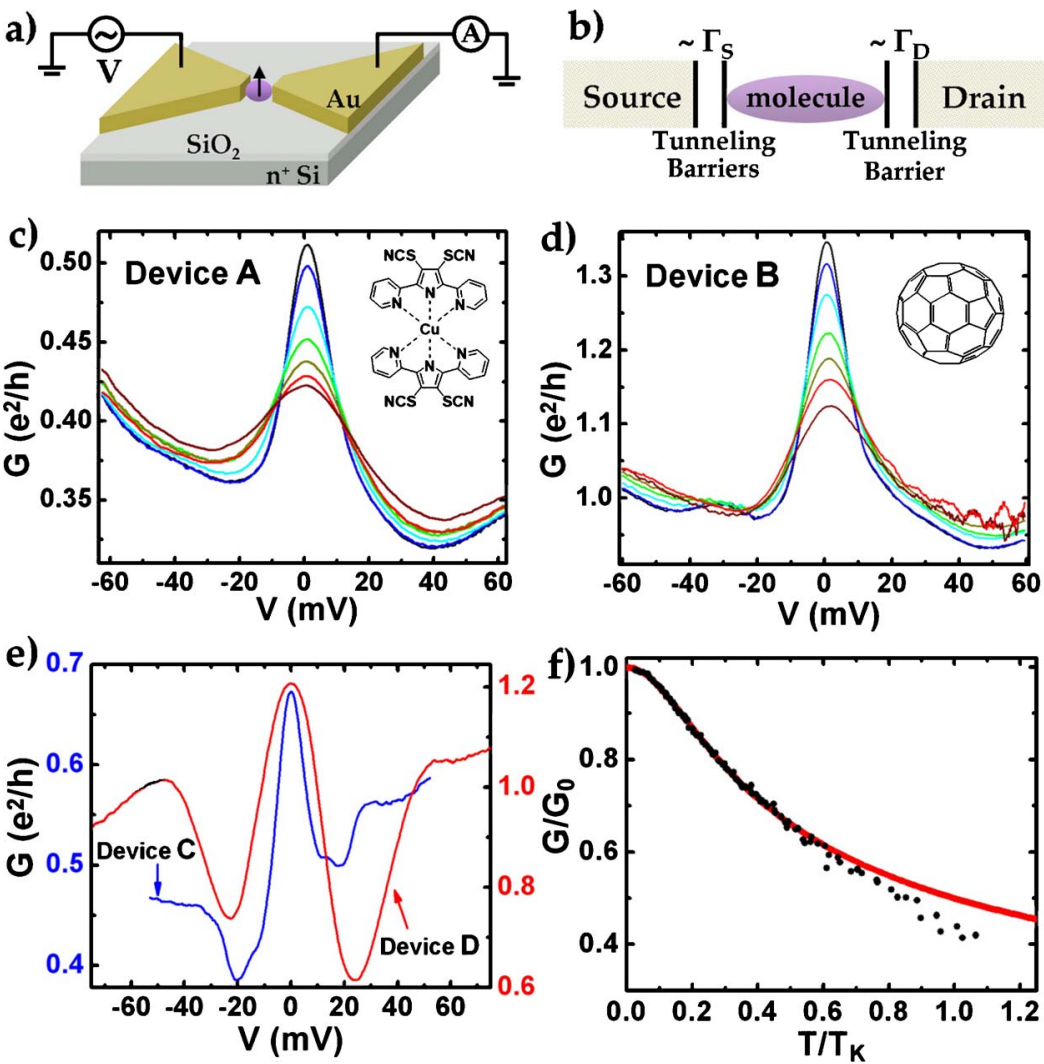

FIG. 1. (Color online) (a) Schematic of SMT measurement setup. (b) Transport path for conduction electrons in SMT device. Traces of $d I / d V$ vs $V$ for several temperatures (c) from $1.8 \mathrm{~K}$ (tallest/black) to $53 \mathrm{~K}$ (shortest/maroon) for device $\mathrm{A}$, using complex 1 , and (d) from $1.8 \mathrm{~K}$ (tallest/black) to $46 \mathrm{~K}$ (shortest/maroon) for device $B$, using $\mathrm{C}_{60}$. Amplitude of Kondo resonance decreases with increasing temperature. Insets: schematic of (c) complex 1 and (d) $\mathrm{C}_{60}$. (e) $d I / d V$ vs $V$ at $T=1.8 \mathrm{~K}$ for two samples, demonstrating the disparate Kondo temperatures associated with different devices. Device C (blue) contains a $\mathrm{C}_{60}$ molecule and $\mathrm{D}$ (red) contains complex 1 . Note that the respective background contributions to the measured conductance have not been subtracted from the plots in (c)-(e). (f) Fit of equilibrium conductance versus temperature for devices A-D, using Eq. (1), yielding $T_{K}$ $\approx 52,43,35$, and $105 \mathrm{~K}$, respectively.

$$
G_{\mathrm{EK}}(T, 0)=G_{0}\left[1+\left(2^{1 / s}-1\right)\left(T / T_{K}\right)^{2}\right]^{-s}+G_{b},
$$

where $s=0.22$ for a spin- $\frac{1}{2}$ impurity. The total conductance comprises the Kondo resonance and a background contribution, $G_{b}$, approximated as linear in applied bias, found via a fit to $d I / d V$ vs $V$ over a bias range that incorporates the conductance minima outside the Kondo resonance. A constant is subtracted from the fit to ensure that $G(T, V)-G_{b}$ $\geq 0$ for all $T$ and $V$. From Eq. (1), $G_{0}$ is the height of the resonant peak in the limit that $T \rightarrow 0 \mathrm{~K}: G_{0}=G(0,0)-G_{b}$. Similarly $T_{K}$ is the temperature at which the Kondo resonance equilibrium conductance is equal to half its zerotemperature value: $G\left(T_{K}, 0\right)-G_{b}=G_{0} / 2$. From this description we extract values of the parameters $T_{K}$ and $G_{0}$ [Fig. 1(f)]. As $T$ increases, deviations from Eq. (1) are expected as higher order transport processes become relevant. Our data are in good agreement with this form up to $T / T_{K}$ $=0.4-0.75$, depending on the device. Further references to $G(T, V)$ denote conductance with the background component removed.

Of the 29 devices analyzed over the full temperature range, 19 incorporated complex 1 , and 10 devices utilized $\mathrm{C}_{60}$, with $T_{K}$ ranging from 34 to $155 \mathrm{~K}$. While the $\mathrm{C}_{60}$-based devices had a lower $T_{K}$ on average $\left(T_{K \text {,av }} \approx 57 \mathrm{~K}\right.$ for $\mathrm{C}_{60}$-based devices compared to $T_{K, \text { av }} \approx 70 \mathrm{~K}$ for devices utilizing complex 1), they were typically less stable with increasing temperature and bias. The enhanced stability of devices made with complex 1 is likely due to their thiocyanate end groups which can covalently bond to the gold electrodes. Irreversible changes in device configuration at higher tem- peratures limited our measurements to the region of $T$ $\lesssim T_{K} / 2$ for the majority of devices.

If the Kondo resonance scales universally with respect to bias and temperature, then we can assume that the scaling function may be approximated by a series expansion. We test this idea by fitting the nonequilibrium conductance to the equation $G(T, V) \approx G_{0}-\widetilde{c}_{T}\left(k_{B} T\right)^{P_{T}}-\widetilde{c}_{V}(e V)^{P_{V}} . P_{V}$ and $P_{T}$ are the scaling exponents for bias and temperature, while $\widetilde{c}_{V}$ and $\widetilde{c}_{T}$ are expansion coefficients. We do not presume the theoretically predicted quadratic form of the scaling exponents and instead estimate $P_{V}$ and $P_{T}$ independently. To extract $P_{V}$ we fit $G(T, 0)-G(T, V)$ to a power-law form in the bias range $|e V| \leq k_{B} T_{K} / 2$ for each trace in which $T \leq T_{K} / 5$. To extract $P_{T}$ this process is repeated for $T \leq T_{K} / 5$ at fixed values of $V$, again in the range $|e V| \leq k_{B} T_{K} / 2$. We limit the $T$ and $V$ ranges because the nonequilibrium conductance is expected to deviate from universal scaling as $k_{B} T$ and $e V$ approach $k_{B} T_{K}$. Averaged over all devices, we find $P_{V}$ $=1.97 \pm 0.047$ and $P_{T}=2.07 \pm 0.18$. Theoretical treatments have found the leading order corrections to the conductance are quadratic in temperature and bias. ${ }^{13,25,26}$ The scaling exponents we find for both $T$ and $V$ are thus very near the predicted value of 2 . Henceforth we use $P_{V}$ and $P_{T}$ equal to 2. The error bars for all extracted parameters in this study represent $95 \%$ confidence intervals.

Following the approach of Grobis et al. ${ }^{19}$ we first obtain a scaling function of normalized temperature, $T / T_{K}$, and bias, $e V / k_{B} T_{K}$, describing the nonequilibrium conductance at low energies. In the context of the Anderson impurity model, ${ }^{11,27}$ the Kondo resonance arises from the local density of states $^{28}$ of a magnetic impurity with charging energy $E_{c}$ which is coupled to the electron bath of the leads; in a dot in 


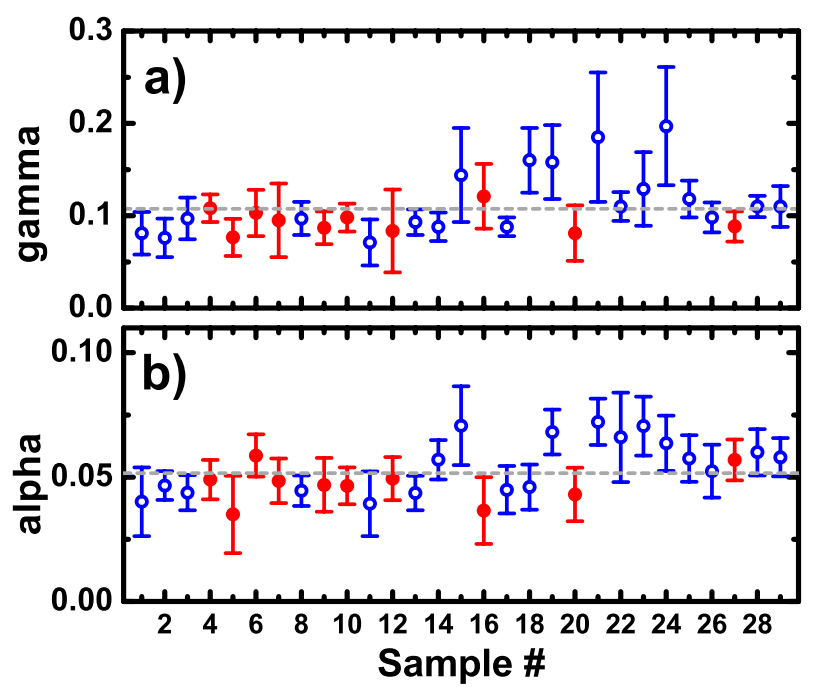

FIG. 2. (Color online) Extracted values of the scaling coefficient (a) $\alpha$ and (b) $\gamma$ for each device. Open blue circles correspond to devices with complex 1 . Filled red circles correspond to devices with $\mathrm{C}_{60}$. Dashed gray line indicates average value. Samples are numbered in order of increasing $T_{K}$.

the Kondo regime this is proportional to the conductance and may be expanded in the low energy limit as ${ }^{19}$

$$
G(T, V)=G_{\mathrm{EK}}(T, 0)\left(1-\frac{c_{T} \alpha}{1+c_{T}\left(\frac{\gamma}{\alpha}-1\right)\left(\frac{T}{T_{K}}\right)^{2}}\left(\frac{e V}{k_{B} T_{K}}\right)^{2}\right),
$$

where $\alpha$ and $\gamma$ are the scaling coefficients we intend to extract. The constant $c_{T} \approx 4.92$. This is set by the low temperature expansion of Eq. (1) in which $G_{\mathrm{EK}}(T, 0) \approx G_{0}[1$ $\left.-c_{T}\left(T / T_{K}\right)^{2}\right]$. The constants $\alpha, \gamma$, and $c_{T}$ are arranged in this fashion so that Eq. (2) can be expanded at low temperature to a simplified power-law form. Here, $\alpha$ and $\gamma$ are defined such that they are independent of the definition of $T_{K}$. Equation (2) in the small $T$ limit is of the form ${ }^{13,14}$

$$
\frac{G(T, 0)-G(T, V)}{c_{T} G_{0}} \approx \alpha\left(\frac{e V}{k_{B} T_{\mathrm{K}}}\right)^{2}-c_{T} \gamma\left(\frac{T}{T_{K}}\right)^{2}\left(\frac{e V}{k_{B} T_{K}}\right)^{2} .
$$

In this expression $\alpha$ represents the $T=0$ curvature of the Kondo resonance with respect to $V$ while $\gamma$ describes the rate at which the resonance peak broadens and decreases in amplitude with increasing temperature.

Figure 2 shows the values of $\alpha$ and $\gamma$ extracted for each sample fitted over the range $T<T_{K} / 5$ and $e V<k_{B} T_{K} / 2$. We calculate average values of $\alpha=0.051 \pm 0.01$ and $\gamma$ $=0.107 \pm 0.027$. Devices with complex 1 had roughly $10 \%$ larger average $\alpha$ and 18\% larger average $\gamma$ compared to $\mathrm{C}_{60}$-based devices. This is due to a few samples containing complex 1 which possess exceptionally large values of $\alpha$ and $\gamma$. These samples may originate from conduction occurring closer to the mixed valence regime, leading to non-Kondo processes preventing an accurate description of the conduc-
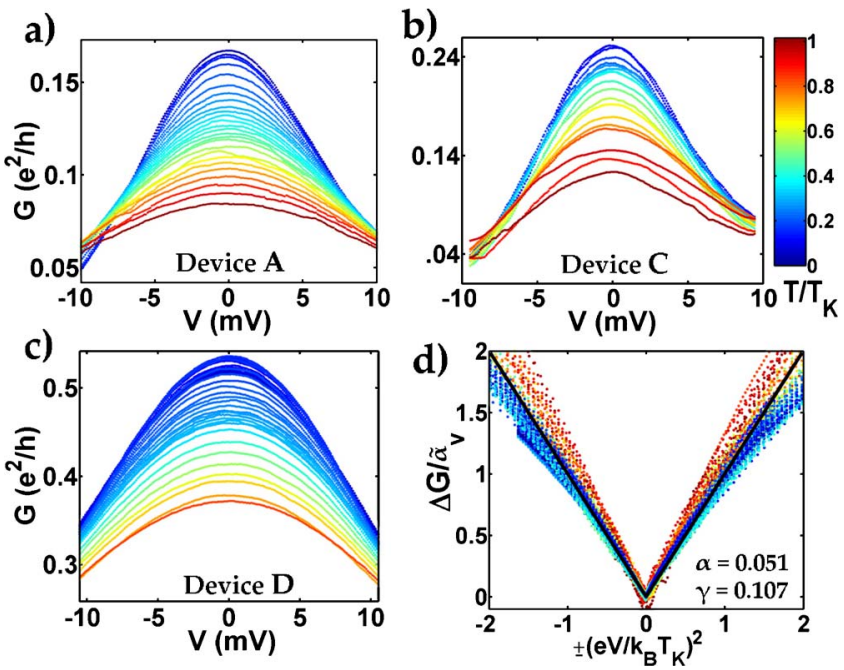

FIG. 3. (Color online) Conductance as a function of $V$ for [(a) and (b) $] T / T_{K} \lesssim 1.0$ and (c) $T / T_{K} \lesssim 0.65$, corresponding to devices A, C, and D from Figs. 1(c) and 1(e), respectively. $G_{b}$ has been subtracted off. (d) Scaled conductance, $\Delta G / \widetilde{\alpha}_{V}$, versus $\left(e V / k_{B} T_{K}\right)^{2}$ for devices A-D, where $\Delta G=[1-G(T, V) / G(T, 0)]$. The data from (a)-(c) are plotted here, along with the analogous data set from device $\mathrm{B}$, using the average extracted values of the scaling coefficients. The solid black line represents the associated universal curve. The color bar in (b) pertains to all four figures.

tance by a universal scaling function. Beyond this minor difference, the extracted coefficients are essentially molecule independent.

Transport measurements $\left[G(T, V)-G_{b}\right.$ vs $\left.V\right]$ in devices A, C, and D from Fig. 1 are shown in Figs. 3(a)-3(c), respectively. In Fig. 3(d) we plot scaled conductance [1 $-G(T, V) / G(T, 0)] / \widetilde{\alpha}_{V}$ versus $\left(e V / k_{B} T_{K}\right)^{2}$ for the data in these figures along with the equivalent set of traces from device $\mathrm{B}$, for $\left(e V / k_{B} T_{K}\right)^{2} \leq 2$ and all available temperatures in the range $T / T_{K} \lesssim 1.0$, using the average extracted values of $\alpha$ and $\gamma$. We define $\widetilde{\alpha}_{V} \equiv c_{T} \alpha /\left[1+c_{T}\left(\frac{\gamma}{\alpha}-1\right)\left(T / T_{K}\right)^{2}\right]$. This plot provides a useful qualitative tool indicating the degree to which transport in the Kondo regime conforms to the predicted scaling function. Data from both devices $\mathrm{A}$ and $\mathrm{B}$ adhere to the scaling form for $\left(e V / k_{B} T_{K}\right)^{2} \lesssim 0.6$ and for $T / T_{K} \lesssim 0.75$, above which deviations exceed the confidence levels. For devices $\mathrm{C} / \mathrm{D}$ the data conform to the scaling function up to $\left(e V / k_{B} T_{K}\right)^{2} \lesssim 0.5 / 0.55$ and $T / T_{K} \lesssim 0.55 / 0.45$. When data for each device are fit to find that device's particular optimal values of $\alpha$ and $\gamma$, the goodness of fit is essentially the same for every device. If we assume the premise of universal scaling such that a single (averaged) $\alpha$ and $\gamma$ should describe all the devices, data from every device with extracted values of $\alpha$ and $\gamma$ that lie within the average error bars collapse onto the scaling curve [black line in Fig. $3(\mathrm{~d})]$ up to at least $\left(e V / k_{B} T_{K}\right)^{2} \lesssim 0.5$ and for $T / T_{K} \lesssim 0.4$.

While the conductance in these devices follows the expected ${ }^{13,14}$ scaling form, we consider the purported universality of the coefficients $\alpha$ and $\gamma$ by examining the level of accord with other experimental data and theoretical expectations. The values extracted from selected experimental measurements of nonequilibrium transport through a GaAs dot 
$\left(T_{K} \approx 0.3 \mathrm{~K}\right)$ with reasonably symmetric coupling $(2: 1)$ indicate $0.1 \leqq \alpha \lesssim 0.15$ and $\gamma \approx 0.5,{ }^{19}$ which deviate from our extracted values beyond the statistical uncertainties in the parameters. Theoretical treatments based on the Anderson ${ }^{11,12,17}$ and Kondo ${ }^{13,14,18,26}$ models focus on defining $\alpha$, which is predicted to take on universal values in the limits of symmetric or strongly asymmetric device coupling. Predictions using various approaches are in the range of $0.1 \lesssim \alpha \lesssim 0.3$, depending on the perturbation method used. Our smaller extracted values of $\alpha$ imply that the Kondo resonances that we observe evolve more slowly than expected with bias voltage given the $T_{K}$ values inferred from their equilibrium temperature dependencies.

There are a number of possible explanations for this systematic difference in $\alpha$. The relative asymmetry of the source and drain coupling may be a relevant issue. The total level broadening $\Gamma=\Gamma_{S}+\Gamma_{D}$, where $\Gamma_{S}$ and $\Gamma_{D}$ are contributions of the respective tunneling barriers as established by the overlap between the local moment and the conduction electron states of the source and drain electrodes, respectively. In a SMT these values are exponentially sensitive to the precise molecule-electrode configuration and will generally be different for every device. We can infer the asymmetry of the coupling from the magnitude of the Kondo resonance as $T$ $\rightarrow 0$, predicted to be $\left(2 e^{2} / h\right)\left(4 \Gamma_{S} \Gamma_{D}\right) /\left(\Gamma_{S}+\Gamma_{D}\right)^{2} \cdot{ }^{29}$ For the SMTs studied here we calculate $\Gamma_{S}: \Gamma_{D}$ ratios between 5.2:1 and 182:1 with a median ratio of approximately 17:1. No systematic difference in coefficient values was found for SMTs with increasing coupling asymmetry ratios. There were also no clear correlations found between scaling coefficient values (and thus deviations from the scaling curve) and values of $T_{K}, G_{0}$, or $G_{b}$.

It is also possible that the molecular character of the devices is relevant to the systematic difference in $\alpha$. The essentially identical observations in $\mathrm{C}_{60}$ and complex 1 devices suggest that there is likely a single explanation that does not depend in detail on molecular structure. This argues against higher-spin Kondo states or orbital degeneracies since such physics would differ significantly between molecule types. Most theoretical treatments assume extremely large (or infinite) on-site repulsions relative to the single-particle level spacing, a relationship that is not necessarily valid in SMTs. Furthermore, molecular devices have vibrational degrees of freedom. ${ }^{9,30}$ These modes have been suggested ${ }^{31,32}$ as relevant to the Kondo regime in SMTs, particularly with respect to the unusually weak gate dependence of the Kondo resonance. ${ }^{22}$ In addition to molecule-specific intramolecular vibrational modes, SMTs possess relatively generic centerof-mass vibrational modes ${ }^{30}$ at low energies $(\sim 5-10 \mathrm{meV})$ that may be relevant across many different molecule types. If the temperature dependence of the Kondo resonance in SMTs is modified by vibrational effects, our extraction of $T_{K}$ values via fitting the temperature dependence may lead to a different effective $\alpha$ than in the bare Kondo system.

We have measured conductance through SMTs in the Kondo regime and found that nonequilibrium transport at low energies is well described by a scaling function of the same functional form as that used for a GaAs quantum dot in the Kondo regime. A quadratic power law in temperature and bias characterized by two scaling coefficients accurately reflects the evolution of the Kondo resonance for SMTs made with either $\mathrm{C}_{60}$ or complex 1 . The extracted values of the scaling coefficients deviate systematically from those in the GaAs case and from theoretical predictions based on idealized models. These deviations highlight the need for further experimental and theoretical investigations of molecular devices and for an improved understanding of the limitations of universality of Kondo physics in realistic nanostructures.

Thanks to Stefan Kirchner, Qimiao Si, and Mike Grobis for useful discussions. D.N. acknowledges support from NSF CAREER under Award No. DMR-0347253 and the David and Lucille Packard Foundation. G.D.S. acknowledges the support of the W. M. Keck Program in Quantum Materials at Rice University. J.M.T. acknowledges support from DARPA.

*gavin.scott@rice.edu

${ }^{1}$ D. Goldhaber-Gordon, H. Shtrikman, D. Mahalu, D. AbuschMagder, U. Meirav, and M. A. Kastner, Nature (London) 391, 156 (1998)

${ }^{2}$ S. M. Cronenwett, T. H. Oosterkamp, and L. P. Kouwenhoven, Science 281, 540 (1998).

${ }^{3}$ J. Nygård, D. H. Cobden, and P. E. Lindelof, Nature (London) 408, 342 (2000).

${ }^{4}$ J. Park, A. N. Pasupathy, J. I. Goldsmith, C. Chang, Y. Yaish, J. R. Petta, M. Rinkoski, J. P. Setna, H. D. Abrũna, P. L. McEuen, and D. C. Ralph, Nature (London) 417, 722 (2002).

${ }^{5}$ W. Liang, M. P. Shores, M. Bockrath, J. R. Long, and H. Park, Nature (London) 417, 725 (2002).

${ }^{6}$ L. H. Yu and D. Natelson, Nano Lett. 4, 79 (2004).

${ }^{7}$ D. Goldhaber-Gordon, J. Göres, M. A. Kastner, H. Shtrikman, D. Mahalu, and U. Meirav, Phys. Rev. Lett. 81, 5225 (1998).

${ }^{8}$ W. G. van der Wiel, S. De Franceschi, T. Fujisawa, J. M. Elzerman, S. Tarucha, and L. P. Kouwenhoven, Science 289, 2105

(2000)

${ }^{9}$ L. H. Yu, Z. K. Keane, J. W. Ciszek, L. Cheng, M. P. Stewart, J. M. Tour, and D. Natelson, Phys. Rev. Lett. 93, 266802 (2004).

${ }^{10}$ N. S. Wingreen and Y. Meir, Phys. Rev. B 49, 11040 (1994).

${ }^{11}$ T. A. Costi, A. C. Hewson, and V. Zlatić, J. Phys.: Condens. Matter 6, 2519 (1994).

${ }^{12}$ R. M. Konik, H. Saleur, and A. Ludwig, Phys. Rev. B 66 , 125304 (2002).

${ }^{13}$ A. Schiller and S. Hershfield, Phys. Rev. B 51, 12896 (1995).

${ }^{14}$ K. Majumdar, A. Schiller, and S. Hershfield, Phys. Rev. B 57, 2991 (1998).

${ }^{15}$ A. Kaminski, Y. V. Nazarov, and L. I. Glazman, Phys. Rev. B 62, 8154 (2000)

${ }^{16}$ A. Rosch, J. Kroha, and P. Wölfle, Phys. Rev. Lett. 87, 156802 (2001).

${ }^{17}$ A. Oguri, J. Phys. Soc. Jpn. 74, 110 (2005).

${ }^{18}$ B. Doyon and N. Andrei, Phys. Rev. B 73, 245326 (2006).

${ }^{19}$ M. Grobis, I. G. Rau, R. M. Potok, H. Shtrikman, and D. 
Goldhaber-Gordon, Phys. Rev. Lett. 100, 246601 (2008).

${ }^{20}$ A. N. Pasupathy, D. C. Bialczak, J. Martinek, J. E. Grose, L. A. K. Donev, P. L. McEuen, and D. C. Ralph, Science 306, 86 (2004).

${ }^{21}$ J. J. Parks, A. R. Champagne, G. R. Hutchison, S. Flores-Torres, H. D. Abrũna, and D. C. Ralph, Phys. Rev. Lett. 99, 026601 (2007).

${ }^{22}$ L. H. Yu, Z. K. Keane, J. W. Ciszek, L. Cheng, J. M. Tour, T. Baruah, M. R. Pederson, and D. Natelson, Phys. Rev. Lett. 95, 256803 (2005).

${ }^{23}$ H. Park, A. K. L. Lim, A. P. Alivisatos, J. Park, and P. L. McEuen, Appl. Phys. Lett. 75, 301 (1999).

${ }^{24}$ D. Natelson, L. H. Yu, J. W. Ciszek, Z. K. Keane, and J. M. Tour,
Chem. Phys. 324, 267 (2006).

${ }^{25}$ P. Nozières, J. Low Temp. Phys. 17, 31 (1974).

${ }^{26}$ M. Pustilnik and L. Glazman, J. Phys.: Condens. Matter 16, R513 (2004).

${ }^{27}$ P. W. Anderson, Phys. Rev. 124, 41 (1961).

${ }^{28}$ K. Nagaoka, T. Jamneala, M. Grobis, and M. F. Crommie, Phys. Rev. Lett. 88, 077205 (2002).

${ }^{29}$ C. W. J. Beenakker, Phys. Rev. B 44, 1646 (1991).

${ }^{30}$ H. Park, J. Park, A. K. L. Lim, E. H. Anderson, A. P. Alivisatos, and P. L. McEuen, Nature (London) 407, 57 (2000).

${ }^{31}$ P. S. Cornaglia, G. Usaj, and C. A. Balseiro, Phys. Rev. B 76, 241403(R) (2007).

${ }^{32}$ F. Elste and F. von Oppen, New J. Phys. 10, 065021 (2008). 